\title{
Arago (1810): the first experimental result against the ether
}

\author{
Rafael Ferrard* \\ Instituto de Astronomía y Física del Espacio, \\ Casilla de Correo 67, Sucursal 28, 1428 Buenos Aires, Argentina \\ and Departamento de Física, Facultad de Ciencias Exactas y Naturales, \\ Universidad de Buenos Aires, Ciudad Universitaria, \\ Pabellón I, 1428 Buenos Aires, Argentina \\ Daniel M. Sforza ${ }^{\dagger}$ \\ Laboratory of Neuro Imaging, Department of Neurology, \\ David Geffen School of Medicine, UCLA, \\ 710 Westwood Plaza, Los Angeles, CA 90095-1769, USA
}

\begin{abstract}
95 years before Special Relativity was born, Arago attempted to detect the absolute motion of the Earth by measuring the deflection of starlight passing through a prism fixed to the Earth. The null result of this experiment gave rise to the Fresnel's hypothesis of an ether partly dragged by a moving substance. In the context of Einstein's Relativity, the sole frame which is privileged in Arago's experiment is the proper frame of the prism, and the null result only says that Snell's law is valid in that frame. We revisit the history of this premature first evidence against the ether theory and calculate the Fresnel's dragging coefficient by applying the Huygens' construction in the frame of the prism. We expose the dissimilar treatment received by the ray and the wave front as an unavoidable consequence of the classical notions of space and time.
\end{abstract}

\section{INTRODUCTION}

Einstein once declared that the Fizeau measurements on the speed of light in moving water "were enough" for experimentally supporting the relativistic notions of space and time. $\frac{1}{-}$ To understand the meaning of this statement, it should be remembered that before 
Einstein's work the lumiferous ether was the supposed material substrate where the luminous perturbation propagates. At first, scientists believed that the ether was not modified by a moving body, but it pervaded all the bodies remaining itself immutable. This property, together with its universality, lent the ether the status of absolute frame (in the newtonian sense). However, in 1818, Augustin Jean Fresnel (1788-1827) $)^{2}$ stated that the ether inside a substance is partly dragged when the substance moves with respect to the (exterior) universal ether. For a transparent substance moving with "absolute" velocity $V$, the ether in the substance moves with "absolute" velocity $\left(1-n^{-2}\right) V$, where $n$ is the refraction index of the substance. The dragging was "measured" in moving water by Fizeau (1851) $\frac{3}{}$ and Michelson and Morley (1886) $\stackrel{4.5}{=}$ Special Relativity abolished the concept of ether in 1905, and these speed measurements are no longer explained as the result of partial dragging but as a relativistic composition of velocities. This is the reason for Einstein's assertion.

The partial dragging of ether is mentioned in the textbooks in connection with the experiments performed by Hoek $(1868)^{\underline{6}}$ and Airy $(1871)^{\underline{7}}$. These experiments integrate the series of attempts to detect the absolute motion of the Earth, which culminated with the crucial Michelson-Morley experiment of $1887^{\frac{8}{\underline{ }}}$ and its repetitions ${ }^{\underline{9}}$. Differing from MichelsonMorley's array, Hoek and Airy forced the light to pass by water to succeed in getting a device which was sensible to the first order in $V / c$. Since Hoek and Airy did not detect any motion, the pre-relativistic Physics justified the null result by resorting to the Fresnel partial dragging of ether. In fact, when the dragging enters into the scene, the effects of first order in $V / c$ cancel out and the expected results turn out to be of a larger order. Thus the absolute motion of the Earth could be undetectable due to the inaccuracy of the experimental devices. This argument helped to keep ether theories sustainable for a time.

It would be completely remarkable that a property of the ether conceived by Fresnel in 1818 could play, 50 years later, a so important role in supporting the ether theory. However it should be said that, far from being visionary, Fresnel's hypothesis was formulated to explain an already existent null result obtained by Arago in $1810^{\underline{10}}$ (for a historical analysis of Fresnel's ether dragging theory see Ref. 11). Dominique François Jean Arago (1786-1853) was a pioneer of the experiments that searched for differences in the speed of light. Arago considered that light coming from different sources would have different velocities. In the framework of the corpuscular model, on which Arago based his arguments and experiments, these differences could be due to diverse reasons: the velocity of the source could influence 
the speed of the emitted light (emission theories), the speed of starlight could be affected by the gravitational field of the star, etc. Arago remarked that Bradley aberration $\frac{12}{\underline{2}}$ was not adequate to detect differences of speed in starlight (a difference of 1/20 would produce a change of 1" in the aberration angle, which was inaccessible to the instruments of that time). Instead, Arago noted that such differences could be registered by measuring the deflection of starlight by a prism: "...by remembering that the deviation experienced by the luminous rays when they obliquely penetrate the diaphanous bodies is a determined function of their original velocity, it can be understood that the observation of the total deviation to which they are subjected when they go through a prism, provides a natural measure of their velocities" ${ }^{13}$ Arago did not succeed in detecting different speeds of light, but he soon realized that the method could be applied to make evident the motion of the Earth: "...when the refractive bodies are in motion, the refraction experienced by a ray should not be calculated with its absolute velocity, but with this same velocity increased or diminished by the one of the body, i.e. with the relative velocity of the ray. ... I devoted myself, in my experiments, to become evident the differences that have to result from the orbital motion of the Earth..." $\underline{\underline{13}}$ The absolute motion referred to by Arago in the former sentences is the motion in the newtonian absolute space; Arago does not mention the ether in his work because it has no place in the corpuscular model.

Arago's experiment was unable to exhibit the motion of the Earth, and Fresnel elaborated an hypothesis to explain this null result in the context of the wave theory of light with an elastic ether. Given the historic importance of Arago's experiment as the first attempt to detect the absolute motion of the Earth and the trigger for the Fresnel hypothesis, the lack of references to Arago's work in the textbooks is intriguing, where only tangential mentions

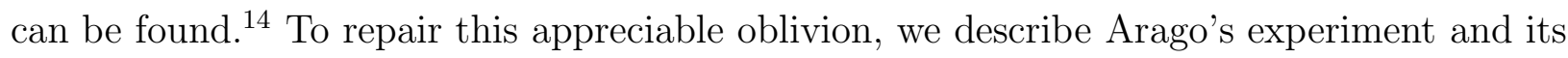
results in Section II. Section III is devoted to revisit the Fresnel hypothesis. In Section IV, we show how the Fresnel partial dragging of ether can explain the null result of Arago's experiment. We do not follow here the demonstration given by Fresnel, who analyzes the deviation in the frame of the universal ether by computing the time of traveling of the rays. Instead we propose to study the envelope of the (anisotropic) secondary wave fronts in the frame of the prism. In this way, we expose the unacceptable dissimilar treatment received by the ray and the wave front, otherwise hidden in Fresnel's original deduction. 


\section{ARAGO'S EXPERIMENT}

As was already mentioned, Arago thought that the refraction of a light ray by a prism depended on the velocity of the prism. Specifically, Arago stated that only the velocity of light relative to the prism should enter the refraction law. Thus, since the prism moves together with the Earth, a starlight ray would suffer different deviations depending on the direction of the ray with respect to the motion of the Earth. These differences would turn out to be maximal between rays traveling in the same direction as the Earth and rays traveling in the opposite direction.

Arago mounted an achromatic prism on the objective lens of a telescope, and he observed several stars through the prism. By comparing this apparent position with the real position of each star, Arago determined how the prism deviated the light rays coming from each star. He expected different angles of deviation for different stars. However, Arago declared: "the rays of all stars are subjected to the same deviations; the slight differences found do not follow any law" 13

Arago made the measurements in two different times of the year. On March 19 and March 27 , he utilized a prism of $24^{\circ}$ and a mural telescope to measure the distances from the stars up to the zenith. On October 8, Arago used a larger prism to cover a half of the objective lens of an improved telescope (namely a repeating circle), which permitted more precise measurements. In this way, the telescope could receive the starlight directly from the star or previously deviated by the prism (the angle between these two different directions, corrected by the time elapsed between both measurements, was the deviation angle). The results are reproduced in Tables [1 [I] and III (the measurements are displayed in the way Arago did it; besides we added the approximated times when the stars passed by the Paris meridian to help in locating the stars in the sky, as Fig. 11 shows). The motion of the earth around the Sun $(30 \mathrm{~km} / \mathrm{s})$ was enough to generate meaningful differences in the deviation angle of stars passing the meridian at 6.00 a.m. and stars passing it at 6.00 p.m. In the first case, the starlight is opposite to the motion of the Earth; in the second one, the starlight goes in the same direction than the Earth. For these two extreme cases, Arago expected differences of 12 " with the prism of $24^{\circ}$ used in March, and 28" in the measurements performed in October. However, there were no traces of any composition of motion entering Snell's law in the results obtained by Arago. 
Arago convinced himself that the only possible interpretation of his null result, in the context of the corpuscular model, was that the sources emit light with all sort of velocities. But our eyes are only sensitive to a narrow band of them. Thus, we are always detecting the same kind of corpuscles, and no differences can be found in their refraction. Concerning the wave theory of light, Arago said that "the explanation for the refraction in this theory is based on a simple hypothesis that is very difficult to submit to calculation. Therefore, I cannot determine in a precise way whether the velocity of the refractive body has some influence on the refraction..."

\section{FRESNEL'S HYPOTHESIS}

In 1818, Fresnel published a letter addressed to Arago, $\frac{2}{2}$ where he discusses the interaction between the ether and the bodies. To begin with, Fresnel mentions that Arago's null result could be easily understood in the context of the wave theory of light by accepting that the Earth imparts its motion to the surrounding ether. In that case, the prism would be at rest in the local ether, and no differences of velocities would appear (the speed of light is a property of the ether). However, in spite of its simplicity, this hypothesis would hinder the understanding of the starlight aberration: "So far I could not conceive this phenomenon the starlight aberration], apart from supposing that the ether freely passes through the globe, and that the velocity imparted to this subtle fluid is nothing but a small part of the one of the Earth, which does not exceed the hundredth for example."15.16 Although the Earth should be pervaded by an ether flow, Fresnel says that light, which is an ether vibration, does not propagate inside the Earth due to an interference of secondary waves. Concerning the transparent media, Fresnel says: "it is evident that the placing of water among the particles, which favors the propagation of luminous vibrations, must be a little obstacle to the establishment of an ether flow." 15 So, Fresnel thought that the way the ether flows through a body depends on the properties of the body.

In contrast to the corpuscular model, Snell's law implies for the wave theory of light that, the bigger the refractive index of a transparent substance, the slower the light propagates in its interior $\underline{\underline{19}}$ Fresnel considered the ether as an elastic material. It is well known that the velocity of waves propagating in an elastic material is proportional to $\rho^{-1 / 2}$, where $\rho$ is the density of the material (the ether, in our case). This means that the density of the ether 
should be bigger in water or glass than in air. At this point, Fresnel's hypothesis about the dragging of ether enters the scene: "only a part of this medium [the interior ether] is dragged by our globe, those constituting the excess of its density with respect to the environmental ether." "... when only a part of the medium moves, the velocity of propagation of the waves should be increased just by the velocity of the center of gravity of the system." 15 Since the Earth does not appreciably drag the ether, there is not excess of ether inside the globe. Instead, the ether is in excess inside the prism, as revealed by the lower speed of light in the substance. The excess of ether in the prism is completely dragged when the prism moves. Only the velocity of the center of gravity of the system "environmental ether - excess of ether" is imparted to the speed of light in the prism. Let $\rho^{\prime}$ be the density of ether inside the prism, and $\rho$ the density of the environmental ether. Thus the excess of ether is $\rho^{\prime}-\rho$. If $V$ is the velocity of the prism relative to the environmental ether (absolute velocity of the

prism), then the speed of the center of gravity of the system "environmental ether - excess of ether" is $\left(\rho^{\prime}-\rho\right) V / \rho^{\prime}$ (the excess of ether is completely dragged). This motion is imparted to the light traveling inside the prism:

$$
v_{d r a g}=\left(1-\frac{\rho}{\rho^{\prime}}\right) V=\left(1-n^{-2}\right) V,
$$

the last member being a consequence of the relation between density of the elastic ether and velocity of propagation of the waves. The coefficient $1-n^{-2}$, where $n$ is the refractive index of the transparent substance, is the so called Fresnel dragging coefficient.

\section{RECOVERING SNELL'S LAW IN THE FRAME OF THE PRISM}

Independently on the way of conceiving the partial dragging of ether, the only tangible effect of the dragging is the restoration of Snell's law in the proper frame of the prism. In this Section, we will show how the restoration works. Our treatment will differ from the one followed by Fresnel. Whereas Fresnel studied ray paths in the frame of the environmental ether (where the prism moves), we instead will use the Huygens' construction in the proper frame of the prism. Although the calculation will follow the precepts of classical Physics, for later discussions we want to control rays and wave fronts in the sole frame that is regarded as privileged in Special Relativity. Like Fresnel, we will concentrate on the simplest case where the incident wave normally strikes the face of the prism and the velocity of the prism 
$\mathbf{V}$ is parallel to this incident direction. In this way the first face does not play any role.

If the ether were not dragged by the prism, the speed of light inside the prism would be $\mathbf{c} / n$ in the frame of the ether and $\mathbf{c} / n-\mathbf{V}$ in the frame of the prism (Galilean composition of velocities). However if the prism partly drags the ether, then the respective velocities change to $\mathbf{c} / n+f \mathbf{V}$ and $\mathbf{c} / n-\mathbf{V}+f \mathbf{V}$, where $f<1$ is a coefficient that quantifies the magnitude of the dragging. According to the Huygens' construction, when the plane wave front traveling inside the prism reaches the second face, each point of this face becomes a secondary emitter. The emerging front wave is given by the envelope of the secondary wave fronts, and the rays go from the secondary emitters to the points where the envelope touch the secondary wave fronts (see Fig. 22). Like Fresnel, we will demand the restoration of Snell's law at the lowest order in $V / c$. For this, we must choose $f$ in such a way that the ray direction does not depend on $V$ at that order. We expect to find $f=1-n^{-2}$.

In order to compute the envelope, we should pay attention to the fact that secondary wave fronts are not spheres in the frame of the prism. This is due to the ether wind that generates an anisotropy in the wave propagation. Concretely, if $\mathbf{k} \cdot \mathbf{r}-\omega t$ is the invariant phase of a wave in the frame of the ether, then, by Galileo transforming the coordinates to a frame moving with velocity $\mathbf{V}$, we obtain:

$$
\mathbf{k} \cdot \mathbf{r}-\omega t=|\mathbf{k}|(\hat{\mathbf{n}} \cdot \mathbf{r}-c t)=|\mathbf{k}|\left(\hat{\mathbf{n}} \cdot\left(\mathbf{r}^{\prime}+\mathbf{V} t\right)-c t\right)=|\mathbf{k}|\left(\hat{\mathbf{n}} \cdot \mathbf{r}^{\prime}-(c-\hat{\mathbf{n}} \cdot \mathbf{V}) t\right),
$$

where $\hat{\mathbf{n}}$ is the direction of propagation. So the phase velocity in a frame moving with velocity $\mathbf{V}$ relative to the ether is

$$
v_{\text {phase }}^{\prime}=c-\hat{\mathbf{n}} \cdot \mathbf{V} \text {. }
$$

This means that a wave front emitted by a point-like source at the coordinate origin is not a sphere in a frame which moves with (absolute) velocity $\mathbf{V}$, but is described by the parametric equations (see Fig. 21)

$$
\begin{aligned}
& x(\delta)=\cos \delta(c-V \cos \delta) t \\
& y(\delta)=\sin \delta(c-V \cos \delta) t
\end{aligned}
$$

where $\mathbf{V}=V \hat{\mathbf{x}}$, and $\hat{\mathbf{n}}=\cos \delta \hat{\mathbf{x}}+\sin \delta \hat{\mathbf{y}}$.

Fig. 2 shows plane wave fronts traveling through the transparent substance with velocity $c / n-(1-f) V$. When a plane front arrives at $\mathrm{O}$, this point becomes an emitter of a 
secondary wave front. After a lapse $t$, the same plane front arrives at E; in the meantime, the secondary wave front emitted at $\mathrm{O}$ has evolved according to Eq. (41). The envelope ER is not orthogonal to the ray OR. Actually the emerging rays are orthogonal to the envelope only in the frame of the ether. Instead, in a moving frame the rays suffer aberration. The deviation angle $\delta$ is the value of the parameter of Eq.(44) such that the straight line passing by E touches one and only one point of each secondary wave front. In general a straight line passing by E satisfies the equation

$$
y=m(x-d)+L=m x+d\left(\frac{1}{\tan \gamma}-m\right)
$$

where $m$ is some slope for the straight line. By replacing $d=(c / n-(1-f) V) t$ and $x$, $y$ by the values given in Eq. (4), one obtains an equation for $\delta(m)$ telling the values of the parameter $\delta$ for which the straight line passing by E intersects the secondary wave front emitted from $\mathrm{O}$. This equation is

$$
\sin \delta(c-V \cos \delta)=m \cos \delta(c-V \cos \delta)+\left(\frac{c}{n}-(1-f) V\right)\left(\frac{1}{\tan \gamma}-m\right)
$$

Since the envelope is tangent to the wave front, we will look for the maximum value of $m$ which makes sense in the Eq. (66). By regarding $m$ as a function of $\delta$, the solution is $\delta$ such that $d m / d \delta=0$. Thus, once Eq. (6) was derivated with respect to $\delta$, one replaces $d m / d \delta=0$ to get

$$
c \cos \delta-V \cos ^{2} \delta+V \sin ^{2} \delta=-m c \sin \delta+2 m V \sin \delta \cos \delta
$$

By keeping only terms which are linear in $V / c$, the slope of the envelope turns out to be

$$
m \simeq-\frac{1}{\sin \delta}\left(\cos \delta+\frac{V}{c}\right)
$$

where now $\delta$ is the direction of the light ray OR. The so obtained value of $m$ can be replaced in Eq. (6) to calculate the direction $\delta$ as a function of the absolute velocity of the substance $V$ and the dragging coefficient $f$. Again we will keep only the terms that are linear in $V / c$. After a bit of algebra, the direction $\delta$ of the emerging light ray results

$$
\frac{1}{\sin \delta}\left(1-\frac{1}{n} \cos \delta\right)-\frac{1}{n \tan \gamma} \simeq\left[\frac{1}{n \sin \delta}-(1-f)\left(\frac{1}{\tan \gamma}+\frac{\cos \delta}{\sin \delta}\right)\right] \frac{V}{c}
$$

Therefore the direction of the emerging ray is not sensitive to the first order in $V / c$-the requirement to explain the null result of the Arago's experiment- when

$$
\frac{1}{n \sin \delta}-(1-f)\left(\frac{1}{\tan \gamma}+\frac{\cos \delta}{\sin \delta}\right)=0
$$


In this case, the direction of the emerging ray fulfills

$$
\frac{1}{\sin \delta}\left(1-\frac{1}{n} \cos \delta\right)-\frac{1}{n \tan \gamma} \simeq 0
$$

i.e., $\sin (\gamma+\delta)=n \sin \gamma$, as it is easily obtained from Snell's law. From the last two equations, we get the Fresnel's dragging coefficient $f=1-n^{-2}$.

\section{CONCLUSIONS}

Fresnel's success in explaining Arago's null results had very important consequences. In the first place, the dominant corpuscular light theory suffered a setback. Arago's null results could not be reasonably understood in the context of the corpuscular model. Thus, the less accepted wave theory of light promoted by Fresnel acquired relevance. The ether dragging theory was an important step to impose the new framework, which became even more accepted after Fresnel's mathematical description of diffraction a few years later (these facts made Arago himself to start supporting the wave theory of light). However the ether theory was not free of problems (for instance, since the refraction indexes of the substances vary with frequency, Fresnel's model would require a different ether for each frequency, which seems implausible), which were eventually solved with its abolition by Special Relativity. Second, the predominance of the wave theory of light put the relationship of ether-absolute motion on the center of the scene.

In Fresnel's wave theory, there is a privileged reference frame (the ether) where light propagates with velocity $c$ in any direction. When a transparent substance is at rest in the ether, Snell's law of refraction is satisfied in the substance. In the case where the substance is moving in the environmental ether, there exists a subtle balance between aberration and partial dragging of the ether whose net result is to hide the absolute motion, because Snell's law remains valid in the frame of the substance in first order in $V / c$. Thus, the ether partial dragging diminishes the privileged status of the ether as the universal frame to which all movements can be referred to.

The partial dragging of ether renders valid Snell's law in the frame of the moving substance in first order in $V / c$, because the ray deviates the same angle in a substance at rest in the ether. However an annoying asymmetry between ray and wave front is left, even in first order in $V / c$ : while the emerging ray is perpendicular to the wave front in the frame of 
the ether, this is not true in the frame of the prism, as a consequence of the anisotropic character of the secondary wave fronts in that frame. In other words, the envelope does not accomplish Snell's law in the frame of the prism, which highlights the incompatibility of Galilean transformations and light theory.

In contrast, Special Relativity states that the only privileged frame in diffraction phenomena is the frame of the refracting substance, and that Snell's law holds exactly true in that reference frame. In addition, the emerging ray (just like any ray propagating in vacuum) is perpendicular to the wave front in any inertial reference system thanks to the relativistic notions of space and time. Thus, the ray and the envelope receive the same treatment. Remarkably, Special Relativity is comfortable with both the corpuscular and wave models of light. From the corpuscular point of view, the aberration of light is a composition of motions. In the context of the wave model, the aberration of light is the change of orientation of the wave front associated with the relativity of the simultaneity of secondary wave emissions.

\section{Acknowledgments}

R.F. was supported by Universidad de Buenos Aires (UBACYT X103) and Consejo Nacional de Investigaciones Científicas y Técnicas (Argentina). We would like to thank Nathan Hageman for reading and commenting on the manuscript. 
* Member of Carrera del Investigador Científico (CONICET, Argentina); Electronic mail: ferraro@iafe.uba.ar

$\dagger$ Electronic mail: sforza@ucla.edu

1 R. S. Shankland, "Conversations with Albert Einstein," Am. J. Phys. 31, 47-57 (1963).

2 A. J. Fresnel, "Lettre de M. Fresnel à M. Arago, sur l'influence du mouvement terrestre dans quelques phénomènes d'optique," Annales de Chimie et de Physique 9, 57-66 (1818).

3 H. Fizeau, "Sur les hypothèses relatives à l'éther lumineux, et sur une expérience qui parait démontrer que le mouvement des corps change la vitesse avec laquelle la lumière se propage dans leur intérieur," Comptes Rendus de l'Académie des Sciences de Paris XXXIII (15), 349355 (1851); "Sur les hypothèses relatives à l'éther lumineux. Et sur une expérience qui parait démontrer que le mouvement des corps change la vitesse avec laquelle la lumière se propage dans leur intérieur," Annales de Chimie et de Physique LVII ( $3^{a}$ série), 385-404 (1859).

4 A. A. Michelson and E. W. Morley, "Influence of motion of the medium on the velocity of light," Am. J. Sci. 31, 377-386 (1886).

5 Fizeau also established that the dragging in moving air was not detectable.

6 M. Hoek, "Determination de la vitesse avec laquelle est entrainée une onde lumineuse traversant un milieu en mouvement," Arch. Néerl. Sci. 3, 180-185 (1868).

7 G. B. Airy, "On a supposed alteration in the amount of astronomical aberration of light, produced by the passage of the light through a considerable thickness of refracting medium," Proc. Roy. Soc. (London) 20, 35-39 (1871).

8 A. A. Michelson and E. W. Morley, "On the Relative Motion of the Earth and the Luminiferous Ether," Am. J. Sci. 34, 333-345 (1887); ibid., "On the Relative Motion of the Earth and the Luminiferous Æther," Philos. Mag. 24, 449-463, (1887).

9 L. S. Swenson, "The Michelson-Morley-Miller experiments before and after 1905," Journal for the History of Astronomy 1, 56-78 (1970).

10 D. F. J. Arago, "Mémoire sur la vitesse de la lumière, lu à la première Classe de l'Institut, le 10 décembre 1810," Comptes Rendus de l'Académie des Sciences XXXVI, 38 -49 (1853); "Vitesse de la lumière (1810)," Euvres Complètes (7), p. 557 (Gide, Paris, 1858).

11 K. M. Pedersen, "Water-filled telescopes and the pre-history of Fresnel's ether dragging," Arch. 
Hist. Exact Sci. 54, 499-564 (2000).

12 "Bradley aberration" or "starlight aberration" is the phenomenon caused by the composition of the (finite) speed of light with the observer motion, which changes the line of sight to the star (observers in relative motion see the same star in different directions). In particular, due to the orbital motion of the Earth, the stars annually trace small ellipses in the sky. J. Bradley, "Account of a new discovered motion of the fixed stars," Phil. Trans. R. Soc. (London) 35, 637-661 (1728).

13 D. F. J. Arago, ibid. (translated by the authors).

14 M. Born, Einstein's Theory of Relativity (Dover, NY, 1962).

15 A. J. Fresnel, ibid. (translated by the authors).

16 Since the rays are perpendicular to the wave front in the ether frame, the starlight aberration should be understood as a complicated process where the wave fronts change their orientation when passing from the universal ether to the local terrestrial ether. A model of this kind was built by Stokes (Ref. 17), but Lorentz found it mechanically inconsistent (Ref. 18).

17 G. G. Stokes, "On the aberration of light," Phil. Mag. (3) 27, 9-15 (1845).

18 H. A. Lorentz, "Over den invloed, dien de beweging der aarde op de lichtverschijnselen uitoefent," Koninklijke Akademie van Wetenschappen (Amsterdam). Afdeeling Natuurkunde. Verslagen en Mededeelingen 2, 297-372 (1885-86). Translated in: "De l'influence du mouvement de la terre sur les phénomènes lumineux," Archives Néerlandaises des Sciences Exactes et Naturelles 21, 103-176 (1887).

19 The speed of light in water was only measured by Foucault in 1850 (Ref. 20), confirming the value $c / n$ ( $n$ is the refractive index) of the wave theory.

20 J. Foucault, "Méthode générale pour mesurer la vitesse de la lumière dans l'air et les milieux transparents. Vitesses relatives de la lumière dans l'air et dans l'eau. Projet d'expérience sur la vitesse de propagation du calorique rayonnant," Comptes Rendus de l'Académie des Sciences de Paris XXX, 551-560 (1850). 
TABLE I: Deviations on March 19, 1810

\begin{tabular}{|c|c|c|}
\hline Time & Star Name & Measured Deviation \\
\hline $18: 10$ & Rigel & $10^{\circ} 4^{\prime} 24^{\prime \prime}, 16$ \\
\hline $18: 50$ & $\alpha$ Orion & $\ldots 25 ", \quad 5$ \\
\hline $20: 28$ & Castor & $\ldots 24^{\prime \prime}, \quad 6$ \\
\hline $20: 35$ & Procyon & $\ldots 24 ", \quad 9$ \\
\hline $20: 38$ & Pollux & $\ldots 29 ", \quad 3$ \\
\hline $22: 23$ & $\alpha$ Hydra & $\ldots 22 ", \quad 6$ \\
\hline 23:02 & Regulus & $\ldots 25 ", \quad 2$ \\
\hline $02: 19$ & Spica & $\ldots 21 ", \quad 4$ \\
\hline 04:30 & $\alpha$ Corona Borealis & $\ldots 22 ", \quad 8$ \\
\hline $04: 38$ & $\alpha$ Serpens & $\ldots 22^{\prime \prime}, \quad 3$ \\
\hline $05: 22$ & Antares & $\ldots 22 ", \quad 5$ \\
\hline 05:30 & $\zeta$ Ophiuchus & $\ldots 24 ", \quad 0$ \\
\hline
\end{tabular}


TABLE II: Deviations on March 27, 1810

\begin{tabular}{lcc}
\hline \hline Time & Star Name & Measured Deviation \\
\hline $18: 18$ & $\alpha$ Orion & $10^{\circ} 4^{\prime} 33^{\prime \prime}, 28$ \\
$19: 55$ & Castor & $\ldots 27^{\prime \prime}, 93$ \\
$20: 02$ & Procyon & $\ldots 32^{\prime \prime}, 31$ \\
$20: 06$ & Pollux & $\ldots 32^{\prime \prime}, 78$ \\
$21: 51$ & $\alpha$ Hydra & $\ldots 28^{\prime \prime}, 32$ \\
$00: 12$ & $\beta$ Leo & $\ldots 30^{\prime \prime}, 21$ \\
$01: 47$ & Spica & $\ldots 26^{\prime \prime}, 29$ \\
$02: 39$ & Arcturus & $\ldots 28^{\prime \prime}, 05$ \\
$03: 58$ & $\alpha$ Corona Borealis & $\ldots 31^{\prime \prime}, 39$ \\
$04: 49$ & Antares & $\ldots 28^{\prime \prime}, 19$ \\
$04: 58$ & $\zeta$ Ophiuchus & $\ldots 29^{\prime \prime}, 64$ \\
$01: 04$ & $\gamma$ Virgo & $\ldots 27^{\prime \prime}, 80$ \\
$01: 18$ & $\delta$ Virgo & $\ldots 27^{\prime \prime}, 34$ \\
$01: 24$ & $\epsilon$ Virgo & $\ldots 31^{\prime \prime}, 42$ \\
$23: 37$ & $\delta$ Leo & $\ldots 34 ”, 02$ \\
\hline
\end{tabular}

TABLE III: Deviations on October 8, 1810

\begin{tabular}{ccc}
\hline \hline Time & Star Name & Measured Deviation \\
\hline $19: 26$ & $\alpha$ Aquila & $22^{\circ} 25^{\prime} 09^{\prime \prime}$ \\
$21: 24$ & Moon crater & $\ldots 25^{\prime} 09^{\prime \prime}$ \\
$21: 40$ & $\alpha$ Aquarius & $\ldots 25^{\prime} 02^{\prime \prime}$ \\
$02: 35$ & $\alpha$ Cetus & $\ldots 25^{\prime} 03^{\prime \prime}$ \\
$04: 08$ & Aldebaran & $\ldots 25^{\prime} 00^{\prime \prime}$ \\
$04: 48$ & Rigel & $\ldots 24^{\prime} 59^{\prime \prime}$ \\
$05: 28$ & $\alpha$ Orion & $\ldots 25^{\prime} 02^{\prime \prime}$ \\
$06: 19$ & Sirius & $\ldots 25^{\prime} 08^{\prime \prime}$ \\
\hline
\end{tabular}




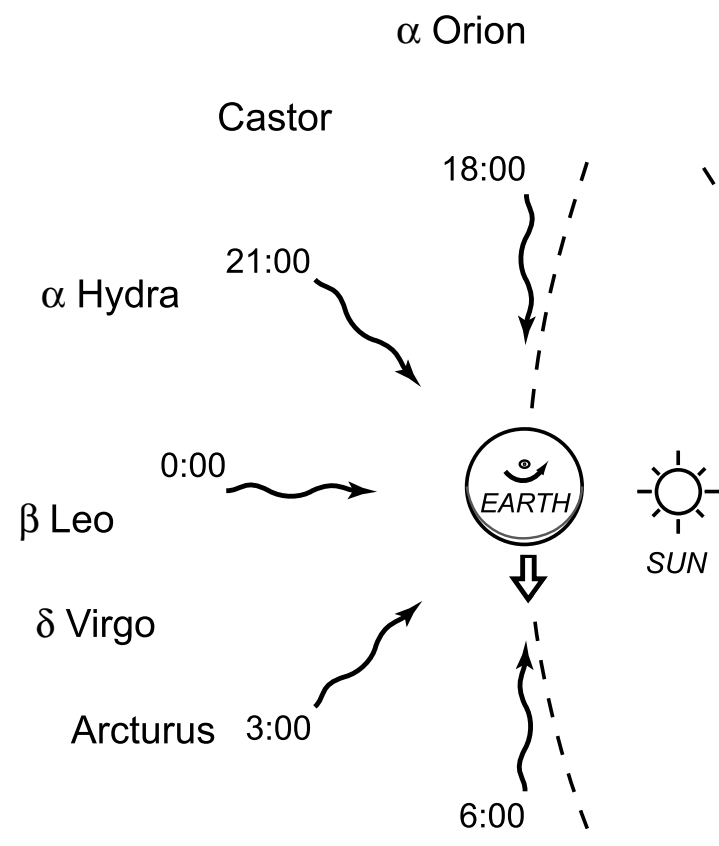

Antares

MARCH OCTOBER

FIG. 1: This diagram shows the light from different stars and the Earth's orbital motion when Arago's measurements were made on March 27 and October 8, 1810. Wavy lines represent light from stars (projected on Earth's orbital plane), and the hollow arrows correspond to the Earth's orbital velocity. The different Galilean compositions of motions were expected to result in different starlight deviations by the prism. 


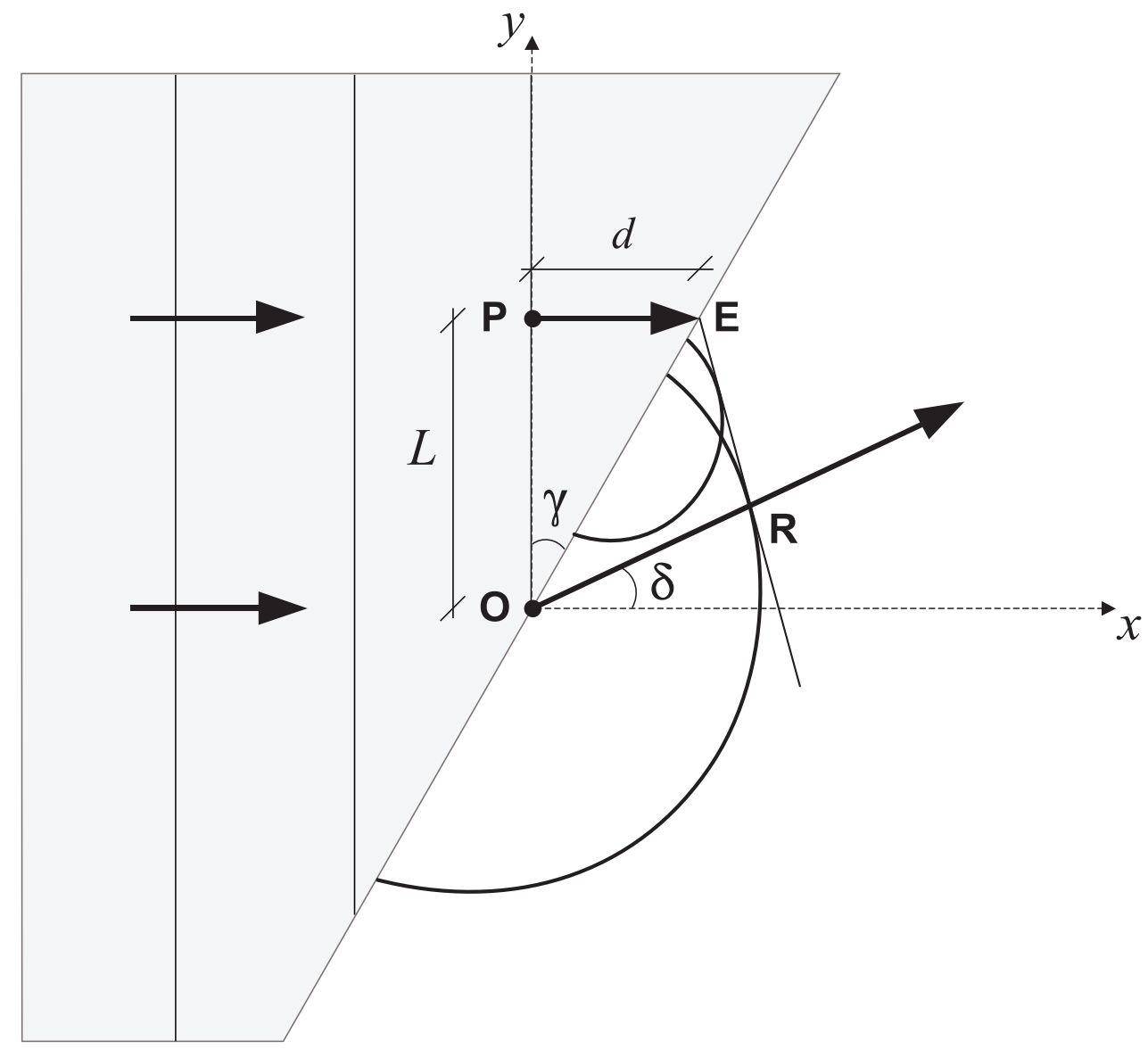

FIG. 2: Huygens' construction in the frame of the prism. 